PROCEEDINGS OF THE

AMERICAN MATHEMATICAL SOCIETY

Volume 138, Number 11, November 2010, Pages 4137-4148

S 0002-9939(2010)10423-9

Article electronically published on June 9, 2010

\title{
INTEGRABILITY CRITERION FOR ABELIAN EXTENSIONS OF LIE GROUPS
}

\author{
PEDRAM HEKMATI \\ (Communicated by Varghese Mathai)
}

\begin{abstract}
We establish a criterion for when an abelian extension of infinitedimensional Lie algebras $\hat{\mathfrak{g}}=\mathfrak{g} \oplus \omega \mathfrak{a}$ integrates to a corresponding Lie group extension $A \hookrightarrow \widehat{G} \rightarrow G$, where $G$ is connected, simply connected and $A \cong \mathfrak{a} / \Gamma$ for some discrete subgroup $\Gamma \subseteq \mathfrak{a}$. When $\pi_{1}(G) \neq 0$, the kernel $A$ is replaced by a central extension $\widehat{A}$ of $\pi_{1}(G)$ by $A$.
\end{abstract}

\section{INTRODUCTION}

Given a group $G$ with a normal subgroup $N$, one may construct the quotient group $H=G / N$. The theory of group extensions addresses the converse problem. Starting with $H$ and $N$, what different groups $G$ can arise containing $N$ as a normal subgroup such that $H \cong G / N$ ? The problem can be formulated for infinitedimensional Lie groups, but the situation is more delicate. Many familiar theorems break down and one must take into account topological obstructions. In particular, Lie's third theorem no longer holds and the question of integrability, i.e. whether a Lie algebra corresponds to a Lie group, becomes relevant 3 .

The aim of this paper is to establish an integrability criterion for abelian extensions of infinite-dimensional Lie groups by generalizing a geometric construction for gauge groups [6, 8, 9, 10, A similar idea is employed in [13] to construct a prequantum bundle for a closed 2 -form with integral periods. For an alternative approach to this problem see [12. In Sections 2 and 3 we review the basic definitions of infinite-dimensional Lie groups and their abelian extensions. Section 4 gives a detailed account of the construction leading up to the integrability criterion.

\section{INFINITE-DIMENSIONAL LIE GROUPS}

We define infinite-dimensional Lie groups along the lines of [11, which should be consulted for further details and for concrete examples. The first step is to define the concept of an infinite-dimensional smooth manifold. Here the bottom line is to replace $\mathbb{R}^{n}$ (or $\mathbb{C}^{n}$ ) by a more general model space on which a meaningful differential calculus can be developed. Essentially all familiar constructions in finite dimensions then carry over to the infinite-dimensional setting. We consider sequentially complete locally convex topological vector spaces. These spaces have

Received by the editors January 20, 2010 and, in revised form, February 2, 2010.

2010 Mathematics Subject Classification. Primary 22E65, 20K35.

Key words and phrases. Infinite-dimensional Lie theory, abelian extensions.

(C)2010 American Mathematical Society

Reverts to public domain 28 years from publication 
the property that every continuous path has a Riemann integral. We adopt the following notion of smoothness.

Definition 2.1. Let $E, F$ be sequentially complete locally convex topological vector spaces over $\mathbb{R}$ (or $\mathbb{C}$ ) and let $f: U \rightarrow F$ be a continuous map on an open subset $U \subseteq E$. Then $f$ is said to be differentiable at $x \in U$ if the directional derivative

$$
d f(x)(v)=\lim _{t \rightarrow 0} \frac{1}{t}(f(x+t v)-f(x))
$$

exists for all $v \in E$. It is of class $C^{1}$ if it is differentiable at all points of $U$ and

$$
d f: U \times E \rightarrow F,(x, v) \mapsto d f(x)(v)
$$

is a continuous map on $U \times E$. Inductively we say that $f$ is of class $C^{n}$ if $d f$ is a map of class $C^{n-1}$ and of class $C^{\infty}$ or smooth if it is of class $C^{n}$ for all $n \geq 1$.

This definition coincides with the alternative notion of convenient smoothness [5] on Fréchet manifolds. A smooth manifold modeled on a sequentially complete locally convex topological vector space $E$ is a Hausdorff topological space $M$ with an atlas of local charts $\left\{\left(U_{i}, \phi_{i}\right)\right\}$ such that the transition functions $\phi_{i} \circ \phi_{j}^{-1}$ : $\phi_{j}\left(U_{i} \cap U_{j}\right) \rightarrow \phi_{i}\left(U_{i} \cap U_{j}\right)$ are smooth on overlaps. A Lie group $G$ is a smooth manifold endowed with a group structure such that the operations of multiplication and inversion are smooth. The Lie algebra $\mathfrak{g}$ is defined as the space of left-invariant vector fields. A vector field $X: G \rightarrow T G$ is left-invariant if

$$
L_{g *} X=X, \forall g \in G
$$

where $L_{g *}$ denotes the pushforward map induced by the diffeomorphism $L_{g}: G \rightarrow$ $G, h \mapsto g h$. By definition $X$ is completely determined by its value at the identity and $\mathfrak{g}$ is therefore identified with $T_{1} G$ as topological vector spaces endowed with the continuous Lie bracket of vector fields. The most striking feature of infinitedimensional Lie theory is that results on existence and uniqueness of ordinary differential equations and the implicit function theorem cease to hold in general beyond Banach Lie groups. Therefore a priori there is no exponential map and even if it exists, it does not have to be locally bijective. The existence and smoothness of the exponential function hinges on the notion of regularity.

Definition 2.2. A Lie group $G$ is called regular if for each $X \in C^{\infty}([0,1], \mathfrak{g})$, there exists $\gamma \in C^{\infty}([0,1], G)$ such that

$$
\gamma^{\prime}(t)=L_{\gamma(t) *}(\mathbf{1}) \cdot X(t), \quad \gamma(0)=\mathbf{1}
$$

and the evolution map

$$
\operatorname{evol}_{G}: C^{\infty}([0,1], \mathfrak{g}) \rightarrow G, \quad X \mapsto \gamma(1)
$$

is smooth.

In other words every smooth curve in the Lie algebra should arise, in a smooth way, as the left logarithmic derivative of a smooth curve in the Lie group. Note that regularity is a sharper condition than the requirement that the exponential map should be defined and smooth. Indeed if $\gamma(t)$ is the curve corresponding to the constant path $X(t)=X_{0}$ for some $X_{0} \in \mathfrak{g}$, then $\gamma(1)=\exp \left(X_{0}\right)$. All known Lie groups modeled on sequentially complete locally convex topological vector spaces are regular [3]. In the convenient setting for calculus, it has been shown [7] that all connected regular abelian Lie groups are of the form $\mathfrak{a} / \Gamma$ for some discrete subgroup 
$\Gamma \subseteq \mathfrak{a}$ of an abelian Lie algebra $\mathfrak{a}$. Moreover, parallel transport exists for connections on principal bundles with regular structure group [4. Important examples of regular Lie groups include gauge groups $C^{\infty}(M, G)$ and diffeomorphism groups $\operatorname{Diff}(M)$, where $M$ is a smooth compact manifold and $G$ is a finite-dimensional Lie group. A Lie group is called locally exponential if the exponential function exists and is a local diffeomorphism at the identity. Gauge groups have this property, but it does not hold true for diffeomorphism groups.

We digress to say a few words about the cohomology of Lie groups and Lie algebras.

2.1. Lie group cohomology. Let $G$ be a Lie group. An abelian Lie group $A$ is called a smooth $G$-module if there is a smooth $G$-action on $A$ by automorphisms $G \times$ $A \rightarrow A,(g, a) \mapsto g . a$. The set of smooth maps $f: G^{n} \rightarrow A$ such that $f\left(g_{1}, \ldots, g_{n}\right)=$ 0 whenever $g_{j}=\mathbf{1}$ for some $j$ are called $n$-cochains and form an abelian group $C^{n}(G, A)$ under pointwise addition. A cochain complex

$$
\cdots \rightarrow C^{n-1}(G, A) \stackrel{\delta_{n-1}}{\longrightarrow} C^{n}(G, A) \stackrel{\delta_{n}}{\longrightarrow} C^{n+1}(G, A) \rightarrow \ldots
$$

is generated by the homomorphisms $\delta_{n}: C^{n}(G, A) \rightarrow C^{n+1}(G, A)$ defined by

$$
\begin{aligned}
\left(\delta_{n} f\right)\left(g_{1}, \ldots, g_{n+1}\right)= & g_{1} . f\left(g_{2}, \ldots, g_{n+1}\right)+\sum_{i=1}^{n}(-1)^{i} f\left(g_{1}, \ldots, g_{i} g_{i+1}, \ldots, g_{n+1}\right) \\
& +(-1)^{n+1} f\left(g_{1}, \ldots, g_{n}\right)
\end{aligned}
$$

and satisfying $\delta_{n} \circ \delta_{n-1}=0$. Let $Z^{n}(G, A)=\operatorname{ker} \delta_{n}$ and $B^{n}(G, A)=\operatorname{im} \delta_{n-1}$ denote the subgroups of $n$-cocycles and $n$-coboundaries respectively. The $n$-th Lie cohomology group is defined by

$$
H^{n}(G, A)=\frac{Z^{n}(G, A)}{B^{n}(G, A)}
$$

2.2. Lie algebra cohomology. Let $\mathfrak{g}$ and $\mathfrak{a}$ be topological Lie algebras. Then $\mathfrak{a}$ is a continuous $\mathfrak{g}$-module if it is abelian and there is a continuous $\mathfrak{g}$-action, $\mathfrak{g} \times \mathfrak{a} \rightarrow$ $\mathfrak{a},(X, v) \mapsto X . v$. Denote by $C^{n}(\mathfrak{g}, \mathfrak{a})$ the vector space of continuous alternating multilinear maps $\omega: \mathfrak{g}^{n} \rightarrow \mathfrak{a}$. A cochain complex

$$
\cdots \rightarrow C^{n-1}(\mathfrak{g}, \mathfrak{a}) \stackrel{d_{n-1}}{\longrightarrow} C^{n}(\mathfrak{g}, \mathfrak{a}) \stackrel{d_{n}}{\longrightarrow} C^{n+1}(\mathfrak{g}, \mathfrak{a}) \rightarrow \ldots
$$

is generated by the linear maps $d_{n}: C^{n}(\mathfrak{g}, \mathfrak{a}) \rightarrow C^{n+1}(\mathfrak{g}, \mathfrak{a})$ given by Palais' formula

$$
\begin{aligned}
\left(d_{n} \omega\right)\left(X_{1}, \ldots, X_{n+1}\right)= & \sum_{i=1}^{n+1}(-1)^{i+1} X_{i} \cdot \omega\left(X_{1}, \stackrel{i}{\cdot}, X_{n+1}\right) \\
& +\sum_{i<j}(-1)^{i+j} \omega\left(\left[X_{i}, X_{j}\right], X_{1}, \stackrel{i}{*} . \stackrel{j}{*}, X_{n+1}\right)
\end{aligned}
$$

and satisfying $d_{n} \circ d_{n-1}=0$. Let $Z^{n}(\mathfrak{g}, \mathfrak{a})=\operatorname{ker} d_{n}$ and $B^{n}(\mathfrak{g}, \mathfrak{a})=\operatorname{im} d_{n-1}$ denote the subspaces of $n$-cocycles and $n$-coboundaries respectively. The $n$-th Lie cohomology group is given by the quotient space

$$
H^{n}(\mathfrak{g}, \mathfrak{a})=\frac{Z^{n}(\mathfrak{g}, \mathfrak{a})}{B^{n}(\mathfrak{g}, \mathfrak{a})} .
$$


In the context of Lie group and Lie algebra extensions, the second cohomology group is important in classifying topologically trivial abelian extensions as we will see. For $n \geq 1$ there is a 'derivation map' $D_{n}: H^{n}(G, A) \rightarrow H^{n}(\mathfrak{g}, \mathfrak{a})$ given by [12]

$$
\left(D_{n} f\right)\left(X_{1}, \ldots, X_{n}\right)=\left.\frac{\partial^{n}}{\partial t_{1} \ldots \partial t_{n}} \sum_{\sigma \in S_{n}} \operatorname{sgn}(\sigma) f\left(\gamma_{\sigma(1)}\left(t_{\sigma(1)}\right), \ldots, \gamma_{\sigma(n)}\left(t_{\sigma(n)}\right)\right)\right|_{t_{i}=0}
$$

where $\gamma_{1}\left(t_{1}\right), \ldots, \gamma_{n}\left(t_{n}\right)$ is any set of smooth curves in $G$ satisfying $\gamma_{i}(0)=\mathbf{1}$ and $\gamma_{i}^{\prime}(0)=X_{i} \in \mathfrak{g}$.

\section{Abelian extensions}

Definition 3.1. An extension of Lie groups is a short exact sequence with smooth homomorphisms

$$
\mathbf{1} \rightarrow A \stackrel{i}{\rightarrow} \widehat{G} \stackrel{p}{\rightarrow} G \rightarrow \mathbf{1}
$$

such that $p$ admits a smooth local section $\sigma: U \rightarrow \widehat{G}, p \circ \sigma=\operatorname{id}_{U}$, where $U \subset G$ is an open identity neighborhood.

The existence of a smooth local section means that $\widehat{G}$ is a principal $A$-bundle over $G$. The extension is called abelian if $A$ is abelian and central if $i(A)$ lies in the center $Z(\widehat{G})$. Two extensions $\widehat{G}_{1}$ and $\widehat{G}_{2}$ are equivalent if there exists a smooth homomorphism $\phi: \widehat{G}_{1} \rightarrow \widehat{G}_{2}$ such that the following diagram commutes:

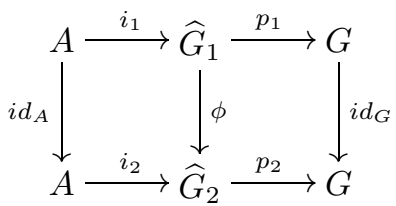

It is straightforward to verify that $\phi$ must be a Lie group isomorphism. The definition for Lie algebras is analogous.

Definition 3.2. An extension of topological Lie algebras is a short exact sequence with continuous homomorphisms

$$
\mathbf{0} \rightarrow \mathfrak{a} \stackrel{i}{\rightarrow} \hat{\mathfrak{g}} \stackrel{p}{\rightarrow} \mathfrak{g} \rightarrow \mathbf{0} .
$$

Two extensions $\hat{\mathfrak{g}}_{1}$ and $\hat{\mathfrak{g}}_{2}$ are said to be equivalent if there is an isomorphism of topological Lie algebras $\phi: \hat{\mathfrak{g}}_{1} \rightarrow \hat{\mathfrak{g}}_{2}$ such that the following diagram commutes:

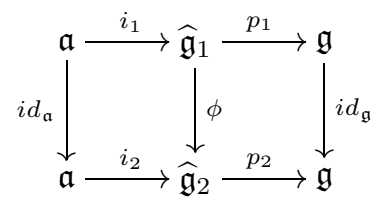

Next we show how abelian extensions can be constructed explicitly. We will assume that as a principal bundle, $\widehat{G}$ is smoothly trivial; i.e. there exists a smooth global section $\sigma: G \rightarrow \widehat{G}$.

Proposition 3.3. Let $G$ be a Lie group, $A$ a smooth $G$-module and $f \in Z^{2}(G, A)$ a 2-cocycle. The smooth manifold $G \times A$ endowed with the multiplication

$$
\left(g_{1}, a_{1}\right)\left(g_{2}, a_{2}\right)=\left(g_{1} g_{2}, a_{1}+g_{1} . a_{2}+f\left(g_{1}, g_{2}\right)\right)
$$

defines an abelian extension $\widehat{G}=G \times_{f} A$ of $G$ by $A$. 
Associativity of the group law follows by the 2-cocycle property. The unit element is $(\mathbf{1}, 0)$ and $(g, a)^{-1}=\left(g^{-1},-g^{-1} \cdot\left(a+f\left(g, g^{-1}\right)\right)\right)$. The extension is smoothly trivial by construction, and the conjugation action of $\widehat{G}$ on $A$ induces the smooth $G$-action. When the cocycle is a coboundary, the extension is isomorphic to the semidirect product $G \ltimes A$. There is a similar cocycle construction for Lie algebras.

Proposition 3.4. Let $\mathfrak{g}$ be a topological Lie algebra, $\mathfrak{a}$ a continuous $\mathfrak{g}$-module and $\omega \in Z^{2}(\mathfrak{g}, \mathfrak{a})$ a 2 -cocycle. The topological vector space $\mathfrak{g} \oplus \mathfrak{a}$ endowed with the continuous Lie bracket

$$
\left[\left(X_{1}, v_{1}\right),\left(X_{2}, v_{2}\right)\right]=\left(\left[X_{1}, X_{2}\right], X_{1} \cdot v_{2}-X_{2} \cdot v_{1}+\omega\left(X_{1}, X_{2}\right)\right)
$$

defines a topologically split abelian extension $\hat{\mathfrak{g}}=\mathfrak{g} \oplus_{\omega} \mathfrak{a}$ of $\mathfrak{g}$ by $\mathfrak{a}$.

It turns out that all smoothly trivial abelian extensions arise in this way. Furthermore, two such extensions are equivalent if and only if the 2-cocycles differ by a 2-coboundary [12, 1 The second cohomology groups $H^{2}(G, A)$ and $H^{2}(\mathfrak{g}, \mathfrak{a})$ therefore parametrize the set of equivalence classes of these extensions. The Lie algebra of $\widehat{G}=G \times_{f} A$ is as one would expect $\hat{\mathfrak{g}}=\mathfrak{g} \oplus_{D_{2} f} \mathfrak{a}$.

Remark 3.5. An abelian extension $\widehat{G}$ is central if and only if $A$ is a trivial $G$-module. Indeed, since the conjugation action of $\widehat{G}$ on $A$ induces the smooth $G$-action, there is for all $g \in G, a \in A$ an element $\hat{g} \in \widehat{G}$ such that $i(g . a)=\hat{g} i(a) \hat{g}^{-1}$. If $i(A) \subset Z(\widehat{G})$, then $i(g . a)=i(a)$ and the injectivity of the inclusion map $i$ implies that $g . a=a$, so the $G$-action is trivial. Conversely, if $G$ acts trivially on $A$, then it follows by $i(a)=\hat{g} i(a) \hat{g}^{-1}$ that $i(A)$ belongs to the center of $\widehat{G}$.

In particular, the extension $\widehat{G}$ is central whenever $G$ is connected and the automorphism group $\operatorname{Aut}(A)$ is discrete. The latter occurs for instance when $A$ is a finite-dimensional real connected abelian Lie group and hence isomorphic to $\mathbb{T}^{m} \times \mathbb{R}^{n}$, where $\mathbb{T}$ is the circle group. Interesting examples of abelian extensions which are non-central arise when $A$ is infinite-dimensional. In 8 Mickelsson describes abelian extensions of gauge groups $C^{\infty}(M, G)$ by the group of circle valued functions $\operatorname{Map}(\mathcal{A}, \mathbb{T})$, where $\mathcal{A}$ denotes the affine space of $\mathfrak{g}$-valued 1 -forms on a compact smooth manifold $M$.

Another interesting case is provided by Neeb, Example 9.16 in [12]: Let $Z$ be an abelian group with the Lie algebra $\mathfrak{z}$. Consider a smooth $Z$-principal bundle $P$ over the compact manifold $M$ together with a connection form $\theta \in \Omega^{1}(P, \mathfrak{z})$. The corresponding curvature form $\omega \in \Omega^{2}(M, \mathfrak{z})$ then defines a 2-cocycle on the Lie algebra of vector fields $\mathcal{V}(M)$. Furthermore, vector fields on $M$ act non-trivially on the commutative gauge algebra $C^{\infty}(M, \mathfrak{z})$ by derivations. The corresponding Lie algebra extension $\widehat{\mathfrak{g}}=\mathcal{V}(M) \oplus_{\omega} C^{\infty}(M, \mathfrak{z})$ is naturally identified with the Lie algebra $\mathcal{V}(P)^{Z}$ of $Z$-invariant vector fields on $P$ and lifts to a non-trivial abelian Lie group extension $C^{\infty}(M, Z) \hookrightarrow \operatorname{Aut}_{Z}(P) \rightarrow \operatorname{Diff}(M)_{0}$.

\section{INTEGRABILITY CRITERION}

In this section we elucidate when an abelian extension of Lie algebras $\hat{\mathfrak{g}}=\mathfrak{g} \oplus_{\omega} \mathfrak{a}$ corresponds to a Lie group extension $\widehat{G}$. If $\omega=D_{2} f$ for some cocycle $f \in Z^{2}(G, A)$,

\footnotetext{
${ }^{1}$ In $\left[12\right.$, the author considers a cohomology theory $H_{s}^{\bullet}(G, A)$ based on locally smooth cocycles. The cohomology groups $H^{n}(G, A)$ in the present paper embed as subgroups in $H_{s}^{n}(G, A)$; cf. Remark 8.5 in 12 .
} 
then by the previous section a corresponding Lie group extension is $\widehat{G}=G \times_{f} A$. In the general case, $\omega$ must satisfy a certain integrality condition that will become apparent by the following construction. The basic idea is to construct $\widehat{G}$ as the quotient of a larger group $\mathcal{P} G \times_{\gamma} A$. This means that in general the extension will be topologically twisted and therefore the group multiplication cannot be described by a smooth global 2-cocycle.

Let $G$ be a connected Lie group of $\mathfrak{g}$ and let $A$ be a smooth $G$-module of the form $\mathfrak{a} / \Gamma$ for some discrete subgroup $\Gamma \subseteq \mathfrak{a}$. We write $e: \mathfrak{a} \rightarrow A$ for the exponential (quotient) map and employ a multiplicative notation. The Lie algebra cocycle $\omega \in Z^{2}(\mathfrak{g}, \mathfrak{a})$ defines a closed $G$-equivariant 2-form $\omega^{e q} \in \Omega^{2}(G, \mathfrak{a})$ by

$$
\omega^{e q}(g)\left(L_{g *} X, L_{g *} Y\right)=\left(L_{g}^{*} \omega^{e q}\right)(\mathbf{1})(X, Y)=g \cdot \omega(X, Y) \quad \forall X, Y \in \mathfrak{g} .
$$

For central extensions, the $G$-action on $A$ is trivial and this is simply the associated left-invariant 2 -form. Let $\mathcal{P} G$ denote the space of smooth based paths $\hat{g}:[0,1] \rightarrow G$ originating at the identity $\hat{g}(0)=\mathbf{1}$ and with some arbitrary endpoint $\hat{g}(1)=g$ in $G$. Given the $C^{\infty}$-topology of uniform convergence of the paths and all their derivatives, $\mathcal{P} G$ becomes a smoothly contractible Fréchet Lie group under pointwise multiplication. It is further a locally trivial $\Omega G$-principal bundle over $G$, where $\Omega G$ is the group of smooth based loops, i.e. smooth paths whose endpoints coincide at the identity [1]. Consider $\mathcal{P} G \times A$ and introduce an equivalence relation

$$
\left(\hat{g}_{1}, a_{1}\right) \sim\left(\hat{g}_{2}, a_{1} e^{\int_{\pi\left[\hat{g}_{1}, \hat{g}_{2}\right]} \omega^{e q}}\right)
$$

whenever two paths with the same endpoint $\hat{g}_{1}(1)=\hat{g}_{2}(1)$ are homotopic and hence form a null homotopic loop. There is then a well-defined 2-dimensional surface $\pi=\pi\left[\hat{g}_{1}, \hat{g}_{2}\right]$ in $G$ bounded by these paths. However, the surface $\pi$ is not unique; it depends on the choice of a smooth homotopy $F:[0,1] \times[0,1] \rightarrow G$ with $F(0, t)=\mathbf{1}$ and $F(1, t)=\left(\hat{g}_{1} * \hat{g}_{2}\right)(t)$, where

$$
\left(\hat{g}_{1} * \hat{g}_{2}\right)(t)= \begin{cases}\hat{g}_{1}(2 t) & 0 \leq t \leq \frac{1}{2} \\ \hat{g}_{2}(2-2 t) & \frac{1}{2} \leq t \leq 1 .\end{cases}
$$

By triangulation $\pi$ can be described by a smooth singular 2-chain. If $\pi^{\prime}$ is another smooth 2-chain with the same boundary, then

$$
e^{\int_{\pi^{\prime}} \omega^{e q}}=e^{\int_{\pi^{\prime}} \omega^{e q}+\int_{\pi+\pi^{-}} \omega^{e q}}=e^{\int_{\pi^{\prime}+\pi^{-}} \omega^{e q}} e^{\int_{\pi} \omega^{e q}}
$$

where $\pi^{-}$denotes $\pi$ with the opposite orientation. Here $\pi^{\prime}+\pi^{-}$is the smooth spherical 2-cycle corresponding to the closed surface obtained by gluing together $\pi^{\prime}$ and $\pi^{-}$along their common boundary. For the equivalence relation to be welldefined we require that $e^{\int_{\pi^{\prime}+\pi^{-}} \omega^{e q}}=\mathbf{1}_{A}$ or equivalently that

$$
\int_{c} \omega^{e q} \in \Gamma
$$

for all spherical cycles $c \in Z_{2}(G)$ [2]. If $c=\partial b \in B_{2}(G)$ is a 2-boundary, then this is automatically satisfied by Stoke's theorem

$$
\int_{\partial b} \omega^{e q}=\int_{b} d \omega^{e q}=0
$$

and therefore the condition factors through to homology. 
We can now proceed to construct the Lie group extension $\hat{G}$ corresponding to $\hat{\mathfrak{g}}=\mathfrak{g} \oplus_{\omega} \mathfrak{a}$ by defining a multiplication on $\mathcal{P} G \times A / \sim$

$$
\left[\left(\hat{g}_{1}, a_{1}\right)\right]\left[\left(\hat{g}_{2}, a_{2}\right)\right]=\left[\left(\hat{g}_{1} \hat{g}_{2}, a_{1}\left(\hat{g}_{1} \cdot a_{2}\right) e^{\gamma\left(\hat{g}_{1}, \hat{g}_{2}\right)}\right)\right]
$$

where $\hat{g} . a:=\hat{g}(1) \cdot a=g . a$ is the given $G$-action on $A$ and $\gamma: \mathcal{P} G \times \mathcal{P} G \rightarrow \mathfrak{a}$ is a smooth 2-cocycle. The latter must be defined in such a way that it yields the correct Lie algebra cocycle $\omega$ and is compatible with the equivalence relation. This is accomplished by choosing

$$
\gamma\left(\hat{g}_{1}, \hat{g}_{2}\right)=\int_{\sigma} \omega^{e q}
$$

where $\sigma: \Delta_{2} \rightarrow G,(t, s) \mapsto \hat{g}_{1}(t) \hat{g}_{2}(s)$ is the smooth singular 2-chain with vertices in $\mathbf{1}, g_{1}$ and $g_{1} g_{2}$ and bounded by the paths $\hat{g}_{1}, g_{1} \hat{g}_{2}$ and $\hat{g}_{1} \hat{g}_{2}$. Here $\Delta_{2}=\{(t, s) \in$ $\left.\mathbb{R}^{2} \mid 0 \leq s \leq t \leq 1\right\}$ is the 2 -simplex in the plane.

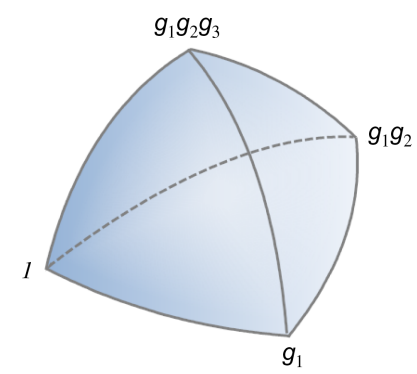

Figure 1. Domain of integration in (4.2).

The 2-cocycle identity

$$
\begin{gathered}
\left(\delta_{2} \gamma\right)\left(\hat{g}_{1}, \hat{g}_{2}, \hat{g}_{3}\right)= \\
=\hat{g}_{1} \cdot \gamma\left(\hat{g}_{2}, \hat{g}_{3}\right)-\gamma\left(\hat{g}_{1} \hat{g}_{2}, \hat{g}_{3}\right)+\gamma\left(\hat{g}_{1}, \hat{g}_{2} \hat{g}_{3}\right)-\gamma\left(\hat{g}_{1}, \hat{g}_{2}\right)=0 \bmod \Gamma
\end{gathered}
$$

is satisfied by (4.1) since the regions of integration form a spherical 2-cycle; see Figure 1 . The face not joining to $\mathbf{1}$ is the left translation by $g_{1}$ of the domain of integration

$$
\hat{g}_{1} \cdot \gamma\left(\hat{g}_{2}, \hat{g}_{3}\right)=\int_{\sigma} g_{1} \cdot \omega^{e q}=\int_{\sigma} L_{g_{1}}^{*} \omega^{e q}=\int_{L_{g_{1}} \sigma} \omega^{e q}
$$

where we have used the $G$-equivariance of $\omega^{e q}$. Thus, we conclude that the multiplication is associative. To see that it is well-defined, i.e. independent of the representatives, a straightforward calculation leads to

$$
\begin{gathered}
\int_{\pi\left[\hat{g}_{1} \hat{g}_{2}, \hat{g}_{1}^{\prime} \hat{g}_{2}\right]} \omega^{e q}-\int_{\pi\left[\hat{g}_{1}, \hat{g}_{1}^{\prime}\right]} \omega^{e q}-\gamma\left(\hat{g}_{1}^{\prime}, \hat{g}_{2}\right)+\gamma\left(\hat{g}_{1}, \hat{g}_{2}\right)=0 \bmod \Gamma, \\
\int_{\pi\left[\hat{g}_{1} \hat{g}_{2}, \hat{g}_{1} \hat{g}_{2}^{\prime}\right]} \omega^{e q}-\int_{\pi\left[\hat{g}_{2}, \hat{g}_{2}^{\prime}\right]} g_{1} \cdot \omega^{e q}-\gamma\left(\hat{g}_{1}, \hat{g}_{2}^{\prime}\right)+\gamma\left(\hat{g}_{1}, \hat{g}_{2}\right)=0 \bmod \Gamma,
\end{gathered}
$$

$\int_{\pi\left[\hat{g}_{1} \hat{g}_{2}, \hat{g}_{1}^{\prime} \hat{g}_{2}^{\prime}\right]} \omega^{e q}-\int_{\pi\left[\hat{g}_{1}, \hat{g}_{1}^{\prime}\right]} \omega^{e q}-\int_{\pi\left[\hat{g}_{2}, \hat{g}_{2}^{\prime}\right]} g_{1} \cdot \omega^{e q}-\gamma\left(\hat{g}_{1}^{\prime}, \hat{g}_{2}^{\prime}\right)+\gamma\left(\hat{g}_{1}, \hat{g}_{2}\right)=0 \bmod \Gamma$. 
Again the regions of integration form closed 2-dimensional surfaces in $G$, depicted in Figure 2. The label on each face refers to the corresponding term in the expressions above, numbered from left to right.
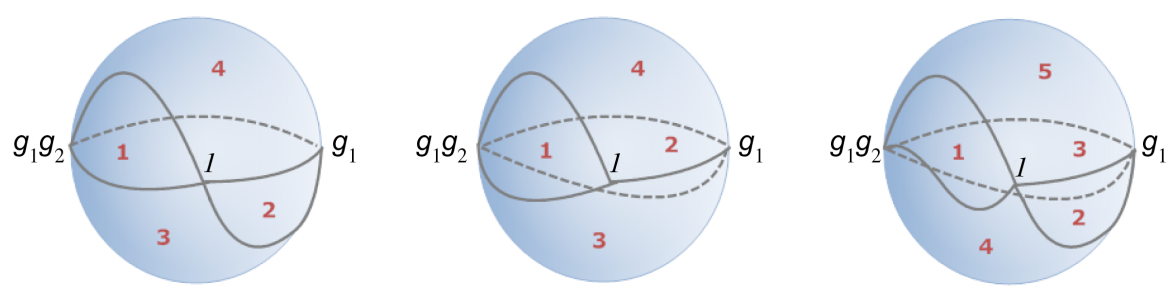

Figure 2. Domain of integration in (4.3), (4.4) and (4.5) respectively.

Next let us calculate the Lie algebra cocycle. We use the equivariance property to evaluate $\omega^{e q}$ only at the identity

$$
\begin{gathered}
\int_{\sigma} \omega^{e q}=\int_{\Delta_{2}} \omega^{e q}(\sigma(t, s))\left(\sigma_{*} \frac{\partial}{\partial t}, \sigma_{*} \frac{\partial}{\partial s}\right) d t \wedge d s \\
=\int_{\Delta_{2}} \omega^{e q}\left(\hat{g}_{1}(t) \hat{g}_{2}(s)\right)\left(\frac{d \hat{g}_{1}(t)}{d t} \hat{g}_{2}(s), \hat{g}_{1}(t) \frac{d \hat{g}_{2}(s)}{d s}\right) d t d s \\
=\left(g_{1} g_{2}\right) \cdot \int_{\Delta_{2}} \omega^{e q}(\mathbf{1})\left(\hat{g}_{2}^{-1}(s) \hat{g}_{1}^{-1}(t) \frac{d \hat{g}_{1}(t)}{d t} \hat{g}_{2}(s), \hat{g}_{2}^{-1}(s) \frac{d \hat{g}_{2}(s)}{d s}\right) d t d s .
\end{gathered}
$$

For any two elements $X, Y \in \mathcal{P} \mathfrak{g}$, we have smooth curves $c_{1}(\tau)=\exp (\tau X(t))$ and $c_{2}(\sigma)=\exp (\sigma Y(s))$ in $\mathcal{P} G$, where exp is defined pointwise by the exponential map of $G$. The Lie algebra cocycle is given by

$$
\begin{gathered}
\left(D_{2} e^{\gamma}\right)(X, Y)=\left.\frac{\partial^{2}}{\partial \sigma \partial \tau}\left(e^{\gamma\left(c_{1}(\tau), c_{2}(\sigma)\right)} e^{-\gamma\left(c_{2}(\sigma), c_{1}(\tau)\right)}\right)\right|_{\tau=\sigma=0} \\
=\frac{\partial^{2}}{\partial \sigma \partial \tau}\left\{(\exp (\tau X(1)) \exp (\sigma Y(1))) \cdot \int_{\Delta_{2}} \omega^{e q}(\mathbf{1})\left(\tau \frac{d X}{d t}, \sigma \frac{d Y}{d s}\right) d t d s\right. \\
\left.-(\exp (\sigma Y(1)) \exp (\tau X(1))) \cdot \int_{\Delta_{2}} \omega^{e q}(\mathbf{1})\left(\sigma \frac{d Y}{d s}, \tau \frac{d X}{d t}\right) d t d s\right\}\left.\right|_{\tau=\sigma=0} \\
=\int_{0 \leq s \leq t \leq 1} \omega^{e q}(\mathbf{1})\left(\frac{d X}{d t}, \frac{d Y}{d s}\right) d t d s+\int_{0 \leq t \leq s \leq 1} \omega^{e q}(\mathbf{1})\left(\frac{d X}{d t}, \frac{d Y}{d s}\right) d t d s \\
=\int_{0 \leq s, t \leq 1} \omega^{e q}(\mathbf{1})\left(\frac{d X}{d t}, \frac{d Y}{d s}\right) d t d s=\omega^{e q}(\mathbf{1})(X(1), Y(1))=\omega(X, Y)
\end{gathered}
$$

where we have used the antisymmetry of $\omega^{e q}$ and the fact that $\omega^{e q}(\mathbf{1})(X(0), Y(0))=$ 0 . Thus, we have verified that $\gamma$ induces a well-defined group multiplication and the correct cocycle at the Lie algebra level. The Lie group extension corresponding to $\hat{\mathfrak{g}}=\mathfrak{g} \oplus_{\omega} \mathfrak{a}$ is the principal $\widehat{A}$-bundle $\widehat{G}=\mathcal{P} G \times_{\gamma} A / \sim \rightarrow G$ with the projection $[(\hat{g}, a)] \mapsto \hat{g}(1)$. If $U \subset G$ is a smoothly contractible open identity neighborhood, then $\sigma: U \rightarrow \widehat{G}, g \mapsto\left[\left(\hat{g}, \mathbf{1}_{A}\right)\right]$ defines a smooth local section, where $\hat{g}$ is the path 
joining 1 and $g$ by a smooth contraction. The fiber $\widehat{A}=\pi_{1}(G) \times_{\gamma} A$ is a central extension of $\pi_{1}(G)$ by $A$. This can be understood by the following argument. If $\pi_{1}(G) \hookrightarrow \tilde{G} \rightarrow G$ denotes the universal covering, then the same construction for $\tilde{G}$ gives rise to the diagram

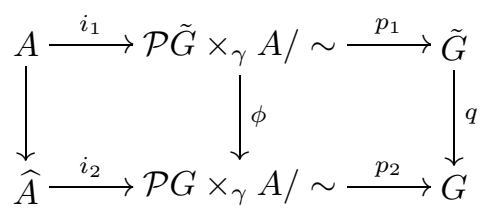

Restriction to the subgroup $\pi_{1}(G) \subset \tilde{G}$ induces a central extension of $\pi_{1}(G)$,

$$
A \stackrel{i_{1}}{\longrightarrow} \pi_{1}(G) \times_{\gamma} A \stackrel{p_{1}}{\longrightarrow} \pi_{1}(G),
$$

since $\pi_{1}(G)$ is discrete and acts trivially on $A$. Finally we have $\widehat{A}=\operatorname{ker} p_{2}=$ ker $q \circ p_{1}=\pi_{1}(G) \times_{\gamma} A$. The right action of the structure group $\widehat{A}$ is given by $[(\hat{g}, a)] .\left[\left(\eta, a^{\prime}\right)\right]=\left[\left(\hat{g} \eta, a\left(\hat{g} \cdot a^{\prime}\right) e^{\gamma(\hat{g}, \eta)}\right)\right]$.

To see that condition (1) is not only sufficient but necessary, let $A \hookrightarrow \widehat{G} \rightarrow G$ be a Lie group extension corresponding to $\hat{\mathfrak{g}}=\mathfrak{g} \oplus_{\omega} \mathfrak{a}$. There is a connection form on this principal bundle whose curvature is the right $G$-equivariant 2 -form $\omega_{R}^{e q} \in \Omega^{2}(G, \mathfrak{a})$,

$$
\omega_{R}^{e q}(g)\left(R_{g *} X, R_{g *} Y\right)=\left(R_{g}^{*} \omega^{e q}\right)(\mathbf{1})(X, Y)=g^{-1} \cdot \omega(X, Y) \quad \forall X, Y \in \mathfrak{g},
$$

where $R_{g}: G \rightarrow G, h \mapsto h g$ denotes the right translation map. Indeed, if $p r_{\mathfrak{a}}$ : $\mathfrak{g} \oplus_{\omega} \mathfrak{a} \rightarrow \mathfrak{a}$ denotes the projection onto the ideal $\mathfrak{a}$, then there is a canonical connection 1-form on $\widehat{G}$ given by

$$
\alpha=\hat{g}^{-1} p r_{\mathfrak{a}}\left(d \hat{g} \hat{g}^{-1}\right) \hat{g} \in \Omega^{1}(\widehat{G}, \mathfrak{a})
$$

with the curvature $\Omega=d \alpha+\frac{1}{2}[\alpha, \alpha]=d \alpha$, where the commutator term vanishes since $\mathfrak{a}$ is abelian. By the Maurer-Cartan equation it follows that

$$
\begin{aligned}
d \alpha & =d \hat{g}^{-1} p r_{\mathfrak{a}}\left(d \hat{g} \hat{g}^{-1}\right) \hat{g}-\hat{g}^{-1} p r_{\mathfrak{a}}\left(d \hat{g} d \hat{g}^{-1}\right) \hat{g}-\hat{g}^{-1} p r_{\mathfrak{a}}\left(d \hat{g} \hat{g}^{-1}\right) d \hat{g} \\
& =-\left[\hat{g}^{-1} d \hat{g}, \hat{g}^{-1} p r_{\mathfrak{a}}\left(d \hat{g} \hat{g}^{-1}\right) \hat{g}\right]+\frac{1}{2} \hat{g}^{-1} p r_{\mathfrak{a}}\left(\left[d \hat{g} \hat{g}^{-1}, d \hat{g} \hat{g}^{-1}\right]\right) \hat{g}
\end{aligned}
$$

Since $\hat{\mathfrak{g}}=\mathfrak{g} \oplus_{\omega} \mathfrak{a}$ is a direct sum, we may write $d \hat{g} \hat{g}^{-1}=\theta_{\mathfrak{g}}+\theta_{\mathfrak{a}}$ as a sum of two 1 -forms, where $\theta_{\mathfrak{g}}$ and $\theta_{\mathfrak{a}}$ only have components along the $\mathfrak{g}$ and $\mathfrak{a}$ directions respectively. We have

$$
\begin{aligned}
d \alpha & =-\left[\hat{g}^{-1} \theta_{\mathfrak{g}} \hat{g}, \hat{g}^{-1} \theta_{\mathfrak{a}} \hat{g}\right]+\hat{g}^{-1} p r_{\mathfrak{a}}\left(\theta_{\mathfrak{g}}^{2}+\theta_{\mathfrak{g}} \theta_{\mathfrak{a}}+\theta_{\mathfrak{a}} \theta_{\mathfrak{g}}+\theta_{\mathfrak{a}}^{2}\right) \hat{g} \\
& =-\hat{g}^{-1} p r_{\mathfrak{a}}\left(\theta_{\mathfrak{g}} \theta_{\mathfrak{a}}+\theta_{\mathfrak{a}} \theta_{\mathfrak{g}}\right) \hat{g}+\hat{g}^{-1} \omega\left(d g g^{-1}, d g g^{-1}\right) \hat{g}+\hat{g}^{-1} p r_{\mathfrak{a}}\left(\theta_{\mathfrak{g}} \theta_{\mathfrak{a}}+\theta_{\mathfrak{a}} \theta_{\mathfrak{g}}\right) \hat{g} \\
& =g^{-1} \cdot \omega\left(d g g^{-1}, d g g^{-1}\right)=\omega_{R}^{e q}(g) .
\end{aligned}
$$

Note that in case of central extensions, there exists a different connection form $\tilde{\alpha}=$ $p r_{\mathfrak{a}}\left(\hat{g}^{-1} d \hat{g}\right)$ on $\widehat{G}$ whose curvature is precisely the left invariant 2-form determined by $\omega$. However, when $G$ acts non-trivially on $A$, the 1 -form $\tilde{\alpha}$ is no longer a connection since it is not invariant under right translation by elements in $A$ and we must therefore use 4.7.

Equipped with a connection, we can define the horizontal lift of a curve on the base space and subsequently the notion of parallel transport. In particular, the 
holonomy around a smooth contractible loop $\eta: \mathbb{T} \rightarrow G$ is given by

$$
\operatorname{hol}(\alpha, \eta)=e^{\int_{\eta} \alpha}=e^{\int_{\pi} \omega_{R}^{e q}}
$$

where $\mathbb{T}=\mathbb{R} / \mathbb{Z}$ denotes the unit circle and $\pi$ is a surface enclosed by $\eta$. The arbitrariness in the choice of this surface leads as before to the requirement $\int_{[c]} \omega_{R}^{e q} \in \Gamma$ for all spherical cycles $[c] \in H_{2}(G)$. The integrality condition $\int_{[c]} \omega^{e q} \in \Gamma$ then follows by the diffeomorphism $g \mapsto g^{-1}$, which relates the right and left $G$-equivariant forms. Moreover, since spherical cycles factor through to spheres, the condition can be formulated equivalently in terms of $\pi_{2}(G) \cong H_{2}(\tilde{G})$. Thus, we are led to the result:

Theorem 4.1 (Integrability criterion). Let $G$ be a connected Lie group and $A$ a smooth $G$-module of the form $\mathfrak{a} / \Gamma$ for some discrete subgroup $\Gamma \subseteq \mathfrak{a}$. The abelian Lie algebra extension $\hat{\mathfrak{g}}=\mathfrak{g} \oplus_{\omega} \mathfrak{a}$ integrates to a Lie group extension $\mathbf{1} \rightarrow \widehat{A} \rightarrow \widehat{G} \rightarrow$ $G \rightarrow \mathbf{1}$ if and only if the image of the period homomorphism

$$
\operatorname{per}_{\omega}: \pi_{2}(G) \rightarrow \mathfrak{a}, \quad[\sigma] \mapsto \int_{[\sigma]} \omega^{e q}
$$

is contained in $\Gamma$, where $\widehat{A}=\pi_{1}(G) \times_{\gamma} A$ is a central extension of $\pi_{1}(G)$ by $A$.

When $G$ is simply connected, we have $\widehat{A}=A$ and the condition coincides with that found in [12]. More generally, whenever the central extension $\widehat{A}$ splits, we may $\bmod$ out by $\pi_{1}(G)$ and the Lie algebra extension lifts to $A \hookrightarrow \widehat{G} \rightarrow G$.

Example 4.2. Consider the two-dimensional torus $G=\mathbb{T}^{2}=\mathbb{R}^{2} / \mathbb{Z}^{2}$ and the left invariant 2 -form corresponding to the symplectic form

$$
\omega=d x \wedge d y
$$

on the universal covering group $\tilde{G}=\mathbb{R}^{2}$. This form is invariant under the $\pi_{1}(G)=$ $\mathbb{Z}^{2}$ action and since $\pi_{2}(G)=0$, all its periods are integral. The central extension $\widehat{\mathfrak{g}}=\mathbb{R}^{2} \oplus_{\omega} \mathbb{R}$ of the Lie algebra $\mathfrak{g}=\mathbb{R}^{2}$ by the trivial $\mathfrak{g}$-module $\mathbb{R}$ defines the Heisenberg group, and according to Theorem 4.1 it lifts to a central extension

$$
\mathbb{Z}^{2} \times_{\gamma} \mathbb{T} \hookrightarrow \widehat{\mathbb{T}^{2}} \rightarrow \mathbb{T}^{2}
$$

In this case, the central extension

$$
\mathbb{T} \hookrightarrow \mathbb{Z}^{2} \times_{\gamma} \mathbb{T} \rightarrow \mathbb{Z}^{2}
$$

does not split and can be characterized as follows. The fundamental group of the torus is the integral lattice in $\mathbb{R}^{2}$, and to each point in the lattice one can associate the path connecting it to the origin, for example, the straight line. For a pair of straight lines $\hat{g}_{1}(t)$ and $\hat{g}_{2}(t)$, the 2-cocycle $\gamma$ is computed as the integral of the 2 -form $\omega$ over a two-dimensional surface determined by the paths. The end result is a 2-cocycle $e^{i \beta(\mathbf{p}, \mathbf{q})}$ for $(\mathbf{p}, \mathbf{q}) \in \mathbb{Z}^{2} \times \mathbb{Z}^{2}$, where $\beta$ is a real antisymmetric bilinear form on the integral lattice. The value of the form $\beta$ is simply the area of the triangle with vertices at $\mathbf{0}, \mathbf{p}$ and $\mathbf{p}+\mathbf{q}$ (up to a normalization of the symplectic form). 
Furthermore, in the universal covering group $\mathbb{R}^{2}$, any two paths with the same endpoints are homotopic, and hence there is no discrete modification of the fibre $\mathbb{T}$ in the corresponding central extension,

$$
\mathbb{T} \hookrightarrow \widehat{\mathbb{R}^{2}} \rightarrow \mathbb{R}^{2}
$$

Remark 4.3. Subsequent to the submission of this paper to the preprint archive arXiv (math/0611431), a similar approach to abelian Lie group extensions using path groups appeared in (math/0703342) [14]. Vizman's idea is to embed the group of contractible loops $\Omega_{0} G \subset \Omega G$ as a normal subgroup in $\mathcal{P} G \times{ }_{\gamma} A$ via the graph of the smooth map

$$
\lambda: \Omega_{0} G \rightarrow A, \quad g \mapsto\left(e^{\int_{\hat{g}} \omega^{e q}}\right)^{-1}
$$

where $\hat{g}$ is an arbitrary path in $\Omega_{0} G$, interpreted as a map $\hat{g}:[0,1]^{2} \rightarrow G$. The quotient group $\mathcal{P} G \times_{\gamma} A / \operatorname{Graph}(\lambda)$ defines an abelian extension of the universal covering group $\tilde{G}$ by $A$. This method is closer in spirit to the constructions in [6] and 9]. Vizman has cited this paper and explained in Remark 8 [14] that the extension $\mathcal{P} G \times_{\gamma} A / \operatorname{Graph}(\lambda)$ is canonically isomorphic to the Lie group $\mathcal{P} \tilde{G} \times_{\gamma} A / \sim$ in diagram (4.6).

\section{ACKNOWLEDGEMENTS}

The author thanks Jouko Mickelsson and Karl-Hermann Neeb for helpful remarks and is grateful to the referees for their thorough reviews and suggestions, which greatly improved the presentation of this paper.

\section{REFERENCES}

1. A. L. Carey and M. K. Murray, String Structures and the Path Fibration of a Group, Comm. Math. Phys. 141, no. 3 (1991), pp. 441-452. MR1134932 (93a:58013)

2. S. Eilenberg, On Spherical Cycles, Bull. Amer. Math. Soc. 47, no. 6 (1941), pp. 432-434. MR 0004778 (3:60e)

3. H. Glöckner, Fundamental Problems in the Theory of Infinite-Dimensional Lie Groups, J. Geom. Symm. Phys. 5 (2006), pp. 24-35. MR2269879 (2007k:22019)

4. A. Kriegl and P. W. Michor, Regular Infinite Dimensional Lie Groups, J. Lie Theory 7 (1997), pp. 61-99. MR1450745 (98k:22081)

5. A. Kriegl and P. W. Michor, The Convenient Setting of Global Analysis, Amer. Math. Soc., Providence, RI (1997). MR.1471480 (98i:58015)

6. A. Losev, G. Moore, N. Nekrasov and S. Shatashvili, Central Extensions of Gauge Groups Revisited, Sel. Math., New Ser. 4(1) (1998), pp. 117-123. MR.1623710 (99k:22031)

7. P. W. Michor and J. Teichmann, Description of Infinite-Dimensional Abelian Regular Lie Groups, J. Lie Theory 9 (1999), pp. 487-489. MR1718235(2000i:22025)

8. J. Mickelsson, Current Algebras and Groups, Plenum Press, New York (1989). MR.1032521 $(90 \mathrm{~m}: 22044)$

9. J. Mickelsson, Kac-Moody Groups, Topology of the Dirac Determinant Bundle and Fermionization, Comm. Math. Phys. 110, no. 2 (1987), pp. 173-183. MR.887993 (89a:22036)

10. M. K. Murray, Another Construction of the Central Extension of the Loop Group, Comm. Math. Phys. 116, no. 1 (1988), pp. 73-80. MR937361 (89e:22035)

11. J. Milnor, Remarks on Infinite-Dimensional Lie Groups, "Relativité, Groupes et Topologie II", B. DeWitt and R. Stora (Eds.), North-Holland, Amsterdam (1983), pp. 1007-1057. MR830252 (87g:22024)

12. K. H. Neeb, Abelian Extensions of Infinite-Dimensional Lie Groups, Mathematical Works. Part XV. Luxembourg: Université du Luxembourg, Séminaire de Mathématique (2004), pp. 69-194. MR2143422(2006i:22022) 
13. A. Pressley and G. Segal, Loop Groups, Clarendon Press, Oxford (1986). MR.900587 (88i:22049)

14. C. Vizman, The Path Group Construction of Lie Group Extensions, J. Geom. Phys. 58, no. 7 (2008), pp. 860-873. MR2426244 (2009f:22015)

Department of Theoretical Physics, Royal Institute of Technology, Albanova UniVersity Center, SE-106 91 Stockholm, Sweden

E-mail address: pedram@kth.se

Current address: School of Mathematical Sciences, University of Adelaide, Adelaide, SA 5005, Australia

E-mail address: pedram.hekmati@adelaide.edu.au 